\title{
Actitudes, hábitos y estrategias de lectura de ingresantes a la educación superior ${ }^{1}$
}

Sindy Patricia Cardona Puello

orcid.org/0ooo-0oo2-9528-4266

Unicolombo, Colombia

scardona@unicolombo.edu.co

\section{Amalfi Herrera Valdez}

orcid.org/0ooo-0oo2-7600-6842

Unicolombo, Colombia

aherrera@unicolombo.edu.co
Alexander Osorio Beleño

orcid.org/oooo-0002-4740-8527

Unicolombo, Colombia

aosorio@unicolombo.edu.co
José Mario González Maza

orcid.org/oooo-0003-4157-410X

Unicolombo, Colombia

jgonzalez@unicolombo.edu.co

\section{Resumen}

El presente artículo muestra el nivel de disposición hacia la lectura que poseen los estudiantes al incursionar en el primer semestre académico de formación universitaria, mediante la identificación de las actitudes, hábitos y estrategias que ellos asumen hacia la lectura. Para obtener los resultados, los autores se basaron en el enfoque de investigación mixta que contempló el diseño y aplicación del test de autorreporte HACES a los estudiantes de primer semestre, matriculados en diferentes programas académicos de la Fundación Universitaria Colombo Internacional en 2017. Para la validación del instrumento se aplicaron técnicas de reducción de datos atendiendo a las condiciones de aplicabilidad para el análisis factorial; asimismo, para medir su fiabilidad se aplicó el coeficiente alfa de Cronbach. Adicionalmente, se utilizó la técnica de grupos focales con el fin de complementar la información recolectada mediante el test. Con este estudio se pudo determinar que los estudiantes, al momento de ingresar a la universidad, reconocen la importancia de la lectura en la formación personal y académica, pero pocos desarrollan un hábito lector y pocos aplican estrategias de lectura adecuadas. Finalmente, se concluye que conocer las actitudes, hábitos y estrategias de lectura permite a las instituciones de educación superior reflexionar sobre la implementación de un plan de formación y fortalecimiento del hábito lector a nivel curricular, acorde con las competencias profesionales exigidas por un mundo globalizado.

$1 \quad$ Este artículo expone los resultados de la investigación "Actitudes, hábitos y estrategias de lectura en estudiantes de primer semestre de la Fundación Universitaria Colombo Internacional-Unicolombo" (Cartagena, Colombia), realizada en 2017.

Recepción: 09/03/2018 | Envío a pares: 17/04/2018 | Aceptación por pares: 24/08/2018 | Aprobación: 28/11/2018 DOI: 10.5294/edu.2018.21.3.6 estudiantes ingresasteis a la educación superior. Educación y Educadores 21(3), 482-503. DOI: 10.5294/edu.2018.21.3.6 


\title{
Palabras clave (fuente: tesauros de la UnEsco)
}

Actitud del estudiante; alfabetización; alfabetización académica; hábito de lectura; lectura; habilidad lectora.

\section{Reading Attitudes, Habits and Strategies of Freshmen ${ }^{1}$}

\begin{abstract}
This article shows the aptitude for reading of first-semester university students. Using mixed research, which included the design and administration of the HACES self-report test to students enrolled in various academic programs of the Fundación Universitaria Colombo Internacional in 2017. Data reduction techniques were used for validation, meeting the applicability conditions for factorial analysis; likewise, Cronbach's alpha coefficient was applied to measure reliability. Additionally, the focal group technique was used to supplement the information collected by the test. It was determined that students, upon entering university, recognize the importance of reading in personal and academic training, but few develop a reading habit and few apply appropriate reading strategies. It is concluded that knowing the reading attitudes, habits and strategies allows higher education institutions to reflect on the implementation of a plan for training and strengthening the reading habit at a curricular level, in accordance with the professional competence demanded by a globalized world.
\end{abstract}

\section{Key words (Source: UnEsco Thesaurus)}

Student attitudes; literacy; academic literacy; reading habit; reading; reading ability.

1 This article presents the results of the research "Reading attitudes, habits and strategies of first-semester students at the Fundación Universitaria Colombo Internacional-Unicolombo" (Cartagena, Colombia) conducted in 2017. 


\section{Atitudes, hábitos e estratégias de leitura de ingressantes na educação superior ${ }^{1}$}

\section{Resumo}

O presente artigo mostra a disposição relacionada à leitura que os estudantes do primeiro semestre acadêmico de formação universitária apresentam. Mediante pesquisa mista, que contemplou a criação e a aplicação do teste de autorreporte HACES, em 2017, aplicou-se o instrumento aos estudantes matriculados em diferentes programas acadêmicos da Fundação Universitária Colombo Internacional em 2017. Para a validação foram usadas técnicas de redução de dados que atendem às condições de aplicabilidade para a análise fatorial; igualmente, para medir sua fiabilidade, aplicou-se o coeficiente alfa de Cronbach. Adicionalmente, utilizou-se a técnica de grupos focais com o objetivo de complementar a informação coletada por meio do teste. Foi possivel determinar que os estudantes, no momento em que entram na universidade, reconhecem a importância da leitura na formação pessoal e acadêmica, mas poucos desenvolvem um hábito leitor e poucos aplicam estratégias de leitura adequadas. Conclui-se que conhecer as atitudes, hábitos e estratégias de leitura permite que as instituições de educação superior reflitam sobre a implementação de um plano de formação e fortalecimento do hábito leitor em âmbito curricular, de acordo com as competências profissionais exigidas por um mundo globalizado.

\section{Palavras-chave (Fonte: Tesauro da UnEsco)}

Atitude do aluno; alfabetização; alfabetização acadêmica; hábito de leitura; leitura; capacidade de leitura. 
La competencia lectora ha sido definida por la Organización para la Cooperación y el Desarrollo Económico (OCDE) como "la comprensión, el uso, la reflexión y el compromiso con los textos escritos, con el fin de alcanzar los propios objetivos, desarrollar el conocimiento y el potencial personales, y participar en la sociedad" (Ministerio de Educación Nacional, 2017, p. 14). Desde esta perspectiva, la lectura constituye una habilidad comunicativa de gran valor para la vida social y profesional, puesto que la construcción, ampliación y circulación del conocimiento exigido por la sociedad contemporánea inician a partir de dicha habilidad. Con independencia de su área de estudio, todo profesional debe poseer la capacidad de leer críticamente textos y discursos de distinta naturaleza; capacidad enunciada igualmente por el Proyecto Tuning (Beneitone et al., 2007) para Europa y América Latina como una competencia genérica común a cualquier titulación. No en vano se considera "que por medio de la lectura los estudiantes del nivel superior entran en contacto con la producción académica propia de una disciplina" (Pérez y Rincón, 2013, p. 136).

Ahora bien, a pesar de la importancia de la lectura para una adecuada interacción comunicativa y un óptimo desenvolvimiento en espacios académicos, en la educación universitaria persisten los problemas relacionados con la competencia lectora, lo que se traduce en un bajo desempeño académico y en serias limitaciones en la formación profesional. Así lo evidenciaron Arista y Paca (2015) en un estudio realizado con estudiantes universitarios de Lengua, Literatura, Psicología y Filosofía en Perú para medir sus hábitos de lectura y niveles de comprensión lectora. Allí observaron, por un lado, que un gran porcentaje de estudiantes poseen hábitos de lectura poco regulares y, por el otro, que el $54 \%$ de los estudiantes encuestados tiene un nivel bajo de comprensión lectora, el $37 \%$ un nivel medio y solo un $9 \%$ presenta un nivel de comprensión lectora alto. En el caso colombiano se puede referenciar, entre otros, el estudio realizado por Calderón y Quijano (2010), en el cual se explica que los estudiantes no poseen hábitos de lectura y no aplican estrategias de comprensión, ya que los libros, las librerías, las bibliotecas y el Internet se asumen como objetos lejanos a su realidad subjetiva y no se incorporan por completo a su praxis profesional disciplinar.

A menudo los docentes universitarios denuncian la renuencia a la lectura y una pobre comprensión lectora de los estudiantes y atribuyen estas dificultades a la formación inadecuada por parte de algunos docentes que manejan una representación utilitarista de la lectura (Molina, 2008), es decir, como instrumento de evaluación. En consecuencia, los estudiantes desarrollan desinterés y apatía hacia la lectura y, por tanto, no alcanzan a formar un hábito lector y no aplican -por desconocimiento o por desidia-estrategias de lectura que conduzcan a la compresión cabal de los textos. Las actitudes, hábitos y estrategias inadecuados limitan el desempeño de los estudiantes universitarios, de allí que sea necesario realizar un seguimiento de dichos aspectos con el fin de reorientar las prácticas de lectura propuestas en la educación superior.

El estudio de las actitudes hacia la lectura cuenta con una estimable tradición en la academia estadounidense y en disciplinas como la psicología social y la pedagogía. Estas se definen como un continuo de sentimientos positivos o negativos y la respectiva predisposición a buscar o evitar actividades que involucren la lectura (Petscher, 2009). Conocer dichas actitudes ayuda a determinar qué aspectos predisponen negativamente a los estudiantes para la lectura y a proponer prácticas dentro del aula para el mejoramiento en este aspecto. Estudiar las actitudes es importante, además, porque "son antecedentes del comportamiento y por lo tanto su estudio se considera prerrequisito para la predicción de la acción" (Parales y Vizcaíno, 2007, p. 353). En esa vía, son varios los autores que le han apostado al diseño de instrumentos de medición de las actitudes hacia la lectura con el fin de determinar qué tipo de acciones se deben adelantar en el aula (Mckenna y Kear, 1990; Wigfield, Guthrie y McGouh, 1996; Con- 
radi, Jang, Bryant, Craft y McKenna, 2013; Mezzalira y Boruchovitch, 2016).

En cuanto al hábito lector, se considera que está regularizado por los gustos e intereses del individuo, por ello se debe considerar como lectora aquella persona que declara leer cualquier tipo de material escrito y si explora prácticas lectoras en soporte impreso o electrónico (Cerlalc, 2011). El deseo de leer nace al asociar la lectura con el placer, la satisfacción y la sensación de logro y entretenimiento; por tanto, el deseo es el factor más poderoso para generar un hábito de lectura que trasciende el imperativo social y el mandato académico o laboral (Salazar, 2006). De igual modo, el hábito lector se entiende como la acción de realizar la lectura en forma automotivada y periódica, por considerarse no solo como una opción de entretención, sino también como medio de aprendizaje, adquisición de nuevo conocimiento y acceso a la cultura ( $\mathrm{Gi}$ lardoni, 2006). En esa medida, en este estudio se considera como lector al sujeto que: a) gusta de la lectura de cualquier tipo de texto o tema; b) lee de manera autónoma, es decir, por iniciativa e interés propios; c) lee textos en cualquier soporte físico, y d) lee de manera habitual o frecuente.

De otro lado, uno de los aspectos que más preocupa en la educación superior es la dificultad de los jóvenes para reconstruir el sentido de un texto y para realizar procesos complejos de pensamiento a partir de lo leído. Esta situación es producto del uso inadecuado de estrategias de lectura, definidas como procedimientos cognitivos y metacognitivos de gran utilidad para regular la actividad lectora, en tanto que permiten reconstruir el sentido global de un texto o hacer frente a los problemas de interpretación que puedan presentarse durante la lectura (Solé, 1998). Para efectos de este estudio, se identificaron distintas estrategias que tienen lugar en momentos diferentes del proceso lector: antes, durante y después de la lectura. En esa medida, se las categorizó en estrategias de aprestamiento, de inmersión, de procesamiento y de metacognición.
Las estrategias de aprestamiento son aquellas que permiten realizar una preparación previa para la lectura de un texto y están relacionadas, según Díaz y Hernández (2010), con el establecimiento del propósito de leer y con las actividades de planificación para afrontar el proceso de comprensión. Las estrategias de inmersión se entienden aquí como todas aquellas acciones tendientes a reconocer la información principal durante la lectura, en otros términos, se refieren al hecho de enfrentarse al contenido del texto e identificar el contenido esencial. Las estrategias de procesamiento son aquellas que permiten comprender lo leído mediante acciones de inferencia o trabajo interpretativo. Las inferencias constituyen, según Díaz y Hernández, el núcleo de la comprensión e implican el empleo de los conocimientos previos para dar contexto y profundidad a la interpretación construida sobre el texto.

Por último, se encuentran las estrategias metacognitivas, las cuales tienen lugar de manera consciente al finalizar la lectura para evaluar la efectividad del proceso de comprensión. Si bien la metacognición puede aparecer durante la lectura, es en la etapa final de la misma cuando los lectores principiantes pueden desplegar, en términos de la pedagoga Jiménez (2004), el "control consciente sobre las destrezas cognitivas". Entre las habilidades de los buenos lectores se encuentra el uso de estrategias metacognitivas como: tomar notas, subrayar, saber qué hacer cuando aparece una palabra desconocida, saber qué leer y cómo leer. Adicionalmente, de acuerdo con Mokhtari y Reichard (2002), las estrategias metacognitivas de lectura pueden entenderse como el conocimiento que tiene el lector de su proceso cognitivo de lectura y como los mecanismos de autocontrol que ejercita cuando monitorea y regula la comprensión textual. El instrumento de medición Metacognitive Awareness of Reading Strategies Inventory (MARSI), diseñado por estos autores en 2002 y revisado en 2018, incluye dentro de las estrategias metacognitivas acciones como la toma de apuntes, la elaboración de resúmenes, esquemas y mapas conceptuales, la formulación de preguntas luego de la lectura, entre otras. 
En Colombia, la preocupación por investigar acerca de la enseñanza de la lectura es reciente. Según explica Gordillo (2015), solo a partir de la década del noventa este se empieza a legitimar como un campo intelectual, gracias a la aparición de la Cátedra Unesco para la Lectura y la Escritura. Un gran número de las investigaciones en el país sobre lectoescritura a nivel universitario están dirigidas a caracterizar las prácticas de lectura y escritura en el aula o a diagnosticar el nivel de comprensión lectora de los estudiantes; no obstante, se registran algunos estudios sobre actitudes, hábitos y estrategias lectoras que, si bien abordan cada factor por separado, constituyen un aporte valioso para el mejoramiento de la competencia comunicativa de los estudiantes y para la construcción de un país de profesionales de comprobada calidad intelectual.

La investigación desarrollada aquí ofreció un perfil de la relación que tienen con la lectura los estudiantes ingresantes a la institución, lo cual permitió pensar en propuestas pedagógicas que contribuyan, por un lado, a superar las posibles deficiencias lectoras y mejorar el desempeño académico de los estudiantes y, por el otro, a la construcción de una comunidad de lectores que responda a las exigencias actuales del mundo globalizado.

Reconocer las actitudes, los hábitos y las estrategias en las prácticas lectoras de los jóvenes es un buen punto de partida para repensar el proceso de enseñanza-aprendizaje en la universidad en lo referente a los procesos de lectoescritura y para cerrar las brechas abiertas durante la educación primaria y secundaria. En la educación media se suelen realizar prácticas de lectura de baja complejidad que no representan un desafío cognitivo para los estudiantes y, por tanto, no permiten afianzar habilidades de lectura crítica e inferencial. A partir de una investigación cualitativa con estudiantes ingresantes a la universidad, Carlino y Fernández (2010) evidenciaron que la mayoría de ellos expresan que en la secundaria las actividades de lectura corresponden a prácticas basadas en modelos memorísticos y transmisivos del conocimiento, lo cual contrasta con la educación superior, en la que la extensión y la cantidad de materiales de lectura es significativamente mayor, sin contar con el nivel de densidad, profundidad y especialización que estos requieren.

\section{Método}

De acuerdo con Hernández, Fernández y Baptista (2014), la naturaleza compleja de los fenómenos de investigación-constituidos por una realidad objetiva y otra subjetiva- representa un factor adicional que ha detonado la necesidad de utilizar los métodos mixtos. Pues bien, las actitudes, hábitos y estrategias de lectura son fenómenos observables a partir de los cuales se puede obtener una información numérica que dé cuenta de las propiedades particulares del fenómeno; sin embargo, se trata de dimensiones complejas que no se agotan solo con la identificación de cifras. Para abordarlas a profundidad se requiere conocer de cerca las apreciaciones que tienen los estudiantes sobre su propio proceso lector, de allí que este estudio posea un enfoque mixto, pues estudia la conducta de un grupo determinado a partir de información de tipo tanto cuantitativo como cualitativo. Según su diseño, este estudio constituye una investigación mixta explicativa secuencial, puesto que -tal como explican Hernández, Fernández y Baptista (2014)- en una primera etapa se recolectaron y analizaron datos cuantitativos y en una segunda fase se recabaron y analizaron datos de carácter cualitativo.

PoBLACIÓN DE ESTUDIO. La fundación Universitaria Colombo Internacional-Unicolombo recibió durante el primer periodo de 2017 un total de 511 estudiantes nuevos provenientes de escuelas públicas y privadas de la ciudad de Cartagena, estudiantes que conforman la población objeto de estudio. Se trabajó con una muestra no probabilística por conveniencia, compuesta por 262 estudiantes ingresantes a los distintos programas de la institución. Al no existir un mecanismo que permita controlar la asistencia de la totalidad de la población ingresante durante la 
semana de inducción, se decidió de antemano trabajar con una muestra, lo que obedece al grado de accesibilidad a los informantes. De dicha muestra, el $57,25 \%$ corresponde a individuos del sexo femenino $y$ el $42,75 \%$ al sexo masculino. El 89,3\% está entre 16-20 años de edad, el 9,9\% entre 21 y 30 años, y el $0,76 \%$ supera los 60 años. Por último, el $76,3 \%$ de los estudiantes de dicha muestra provienen de escuelas públicas y el $23,7 \%$ de escuelas privadas.

El test de autorreporte fue diligenciado en línea, en las instalaciones de la institución, durante la semana de inducción a los nuevos estudiantes. Durante la aplicación se expuso el objetivo de la investigación y se les solicitó a los participantes absoluta honestidad en sus respuestas. Adicionalmente, se usó la técnica de grupo focal para precisar otros factores que permitieran complementar la información arrojada por la encuesta y tener una visión más completa acerca del fenómeno de estudio. Se conformaron dos grupos focales: uno para la jornada de la mañana y otro para la jornada de la tarde, y en contra jornada para garantizar que los estudiantes participaran de manera completamente voluntaria. Cada grupo estuvo conformado por diez estudiantes y moderado por uno de los investigadores, quien propuso los temas a tratar de acuerdo con los objetivos de la investigación.

INSTRUMENTO. Para la recolección y medición de datos se diseñó un test de autorreporte con una escala tipo Likert, denominado test Haces, el cual fue diligenciado en línea durante la semana de inducción a los ingresantes. El diseño de dicho test obedeció a la necesidad de integrar en un mismo instrumento tres dimensiones que habitualmente habían sido estudiadas por separado. Si bien existen cuestionarios estandarizados para medir las actitudes -como The Survey of Adolescent Reading Attitudes (SARA) de Conradi et al., (2013)-y cada país en América Latina diseña una encuesta nacional sobre lectura para medir el hábito lector entre los ciudadanos, no era posible, por razones de tiempo, aplicar a la muestra varios cuestionarios con un alto número de reactivos, ya que podría generar la reticencia de los estudiantes para responderlos en su totalidad.

La validación del test se realizó mediante la aplicación de técnicas de reducción de datos y se verificaron las condiciones previas para el Análisis Factorial Exploratorio (AFE) de la matriz de correlaciones, la matriz anti imagen, el test de esfericidad de Barlett y la medida KMO (Kaiser, Meyer y Olkin); asimismo, para medir su fiabilidad, se aplicó el coeficiente alfa de Cronbach a cada una de las dimensiones. El test fue sometido a una prueba piloto, en noviembre de 2016, con 115 estudiantes de primer semestre de todos los programas de la institución. El pilotaje evidenció la necesidad de incorporar algunas modificaciones al instrumento para aumentar su validez y confiabilidad, tales como la eliminación de tres reactivos en la dimensión de "actitudes", puesto que apuntaban más a lo conductual, con ello la confiabilidad del test aumentó de 0,72 a 0,814.

La versión definitiva del test Haces quedó conformada por tres grandes dimensiones: hábitos, actitudes y estrategias, antecedidas de cuatro preguntas de selección múltiple que indagaron acerca del tiempo dedicado diariamente a la lectura, el espacio y el medio de preferencia: ¿cuánto tiempo dedico a la lectura recreativa diariamente?, ¿cuánto tiempo dedico a la lectura académica diariamente?, ¿en qué lugar prefiero leer? y ¿prefiero realizar la lectura en un medio impreso, digital o en ambos?

La dimensión Hábitos está compuesta por nueve ítems y una escala de frecuencia de 1 a 5 . Esta dimensión presenta un alpha de Cronbach de 0,856, lo que determina su confiabilidad. Aquí se realizó un análisis factorial exploratorio mediante el método de extracción y análisis de componentes principales y con método de rotación varimax. La varianza obtenida fue de 59,385, KMO = 0,884, Sig = 0,000, determinante de 0,030, y en la Prueba de Barlett Chi Cuadrado se obtuvo 387,419. Al aplicar el análisis factorial, se identificaron dos factores, correspondientes a "autonomía lectora" (capacidad de leer por interés e iniciativa propios) y a "recursividad" (capacidad de buscar y obtener los materiales de lectura). 
Tabla 1. Categorización: reactivos por factor en la dimensión Hábitos

\begin{tabular}{|c|c|c|}
\hline Dimensión & Factores & Reactivos \\
\hline \multirow[t]{2}{*}{ Hábitos } & Autonomía lectora & $\begin{array}{l}\text { Hago lecturas adicionales a las suministradas por el docente. } \\
\text { Tengo mis propios libros en casa para leer cuando quiera. } \\
\text { Me llama la atención participar en clubes de lectura. } \\
\text { Hago lecturas de mi propio interés. } \\
\text { La lectura es un hobbie que practico constantemente. } \\
\text { Cuando necesito distraerme busco algo para leer. } \\
\text { En mi tiempo libre leo cuentos, poemas, novelas, etc. }\end{array}$ \\
\hline & Recursividad & $\begin{array}{l}\text { Si no puedo comprar un libro lo pido prestado. } \\
\text { Con frecuencia presto libros en una biblioteca. }\end{array}$ \\
\hline
\end{tabular}

Fuente: elaboración propia.

Para facilitar el análisis de los resultados, se asignó a cada factor una categorización, tal como se muestra en la Tabla 2:

Tabla 2. Categorización de la dimensión Hábitos

\begin{tabular}{|c|c|c|c|}
\hline Dimensión & Factores & Escala & Categoria \\
\hline \multirow{10}{*}{ Hábitos } & \multirow{5}{*}{ Autonomía lectora } & 1. Nada & \multirow{2}{*}{ No lector } \\
\hline & & 2. Poco & \\
\hline & & 3. Medianamente & Lector no autónomo \\
\hline & & 4. Bastante & \multirow{2}{*}{ Lector autónomo } \\
\hline & & 5. Mucho & \\
\hline & \multirow{5}{*}{ Recursividad } & 1. Nada & \multirow{2}{*}{ No recursivo } \\
\hline & & 2. Poco & \\
\hline & & 3. Medianamente & \multirow{2}{*}{ Recursivo } \\
\hline & & 4. Bastante & \\
\hline & & 5. Mucho & Muy recursivo \\
\hline
\end{tabular}

Fuente: elaboración propia.

La dimensión Actitudes está compuesta por nueve ítems y una escala de acuerdo de 1 a 5 . En ella se identificaron dos factores correspondientes a "utilidad de la lectura" (percepciones sobre la importancia o utilidad de la lectura) y "afectividad" (emoción experimentada al momento de leer). Esta dimensión presenta confiabilidad, en la medida que obtuvo un alfa de Cronbach de 0,814. La varianza obtenida fue de $63,415, \mathrm{KMO}=0,881$, Sig $=0,000$, determinante de 0,013, y en la prueba de Barlett Chi Cuadrado $=480,967$. 
Tabla 3. Categorización: reactivos por factor en la dimensión Actitudes

\begin{tabular}{|l|l|l|}
\hline Dimensión & \multicolumn{1}{|c|}{ Factores } & \multicolumn{1}{c|}{ Reactivos } \\
\hline \multirow{5}{*}{ Actitudes } & Utilidad & $\begin{array}{l}\text { La lectura es útil en mi proceso de aprendizaje. } \\
\text { La lectura es útil en mi proceso de formación personal. } \\
\text { La lectura es útil para la vida porque desarrolla las habilidades de } \\
\text { pensamiento (sintetizar, interpretar y argumentar). } \\
\text { El ejercicio de la lectura amplía mi vocabulario. } \\
\text { La lectura enriquece mis conocimientos. } \\
\text { Leer me sirve para ampliar mi comprensión del mundo. }\end{array}$ \\
\cline { 2 - 4 } & Afectividad & $\begin{array}{l}\text { La lectura es una actividad placentera para mí. } \\
\text { La lectura de textos de entretenimiento, como revistas de farándula, } \\
\text { historietas, caricaturas, etc., es divertida. } \\
\text { La lectura de cuentos, poemas, novelas, etc., es atractiva. }\end{array}$ \\
\hline
\end{tabular}

Fuente: elaboración propia.

Cada factor de la dimensión Actitudes se analizó a partir de las categorías que se muestran en la Tabla 4:

Tabla 4. Categorización de la dimensión Actitudes

\begin{tabular}{|c|c|c|c|}
\hline Dimensión & Factores & Escala & Categoria \\
\hline \multirow{10}{*}{ Actitudes } & \multirow{5}{*}{ Utilidad de la lectura } & 1. Nada & \multirow{3}{*}{ Actitud negativa } \\
\hline & & 2. Poco & \\
\hline & & 3. Medianamente & \\
\hline & & 4. Bastante & \multirow{2}{*}{ Actitud positiva } \\
\hline & & 5. Mucho & \\
\hline & \multirow{5}{*}{ Afectividad } & 1. Nada & \multirow{3}{*}{ Actitud negativa } \\
\hline & & 2. Poco & \\
\hline & & 3. Medianamente & \\
\hline & & 4. Bastante & \multirow{2}{*}{ Actitud positiva } \\
\hline & & 5. Mucho & \\
\hline
\end{tabular}

Fuente: elaboración propia.

Por último, la dimensión Estrategias está compuesta por 17 ítems y una escala de frecuencia de 1 a 5 . En ella se identificaron cuatro factores correspondientes a "aprestamiento" (preparación previa para la lectura de un texto); "inmersión" (acciones durante la lectura para reconocer la información principal); "procesamiento" (estrate- gias para comprender lo leído), y “metacognición” (estrategias para regular y evaluar lo aprendido). Posee un alfa de Cronbach de o,918, lo que determina su confiabilidad. La varianza obtenida fue de 66,752, $\mathrm{KMO}=0,886$, Sig $=0,000$, determinante de 0,001 y en la prueba de Barlett Chi Cuadrado $=1745,406$. 
Tabla 5. Categorización: reactivos por factor en la dimensión Estrategias

\begin{tabular}{|l|l|l|}
\hline Dimensión & \multicolumn{1}{|c|}{ Factores } & \multicolumn{1}{c|}{ Reactivos } \\
\hline \multirow{5}{*}{ Estrategias } & Aprestamiento & $\begin{array}{l}\text { Exploro información general sobre el autor antes de leer. } \\
\text { Indago información acerca del texto antes de realizar la lectura. } \\
\text { Establezco con anterioridad el propósito de la lectura. }\end{array}$ \\
\cline { 2 - 4 } & Inmersión & $\begin{array}{l}\text { Resalto las palabras claves durante la lectura. } \\
\text { Subrayo las oraciones principales durante la lectura. }\end{array}$ \\
\cline { 2 - 4 } & $\begin{array}{l}\text { Realizo predicciones sobre el contenido del texto antes de empezar a leer. } \\
\text { Cuando desconozco una palabra durante la lectura, la busco en un diccionario. } \\
\text { Cuando leo una frase difícil de comprender me detengo y releo para hallar el } \\
\text { sentido. } \\
\text { Cuando leo una frase difícil de comprender consulto a otra persona. }\end{array}$ \\
\cline { 2 - 4 } & Metacognición & $\begin{array}{l}\text { Realizo apuntes durante la lectura. } \\
\text { Elaboro resúmenes después de la lectura para una mayor comprensión. } \\
\text { Elaboro esquemas o mapas conceptuales después de la lectura para una mayor } \\
\text { comprensión. } \\
\text { Después de la lectura, busco información en otras fuentes para ampliar lo leido. }\end{array}$ \\
\hline
\end{tabular}

Fuente: elaboración propia.

Cada factor de la dimensión de Estrategias se analizó a partir de las categorías que se muestran en la Tabla 6:

Tabla 6. Categorización de la dimensión de Estrategias

\begin{tabular}{|l|l|l|l|}
\hline \multicolumn{1}{|c|}{ Dimensión } & \multicolumn{1}{|c|}{ Factores } & \multicolumn{1}{c|}{ Cscalas } & Categoria \\
\hline \multirow{4}{*}{ Estrategias } & Aprestamiento & 1. Nunca & \multirow{2}{*}{ Uso ocasional } \\
\cline { 2 - 4 } & Inmersión & 2. A veces & \multirow{2}{*}{ Uso frecuente } \\
\cline { 2 - 3 } & Procesamiento & 3. Regularmente & 4. Casi siempre \\
\cline { 2 - 3 } & Metacognición & 5. Siempre & \\
\cline { 2 - 3 } & & &
\end{tabular}

Fuente: elaboración propia

\section{Resultados}

Uno de los primeros resultados observados es que entre los estudiantes ingresantes hay una tendencia a preferir la lectura recreativa (historietas, textos literarios, libros de autoayuda, revistas, artículos de periódico) en lugar de la lectura académica (artículos de revistas académicas, libros, tesis de grado, entre otros). Así, un poco más de la mitad $(50,4 \%)$ dedica diariamente entre una y dos horas a la lectura recreativa, mientras que $46,9 \%$ dedica el mismo tiempo a la lectura académica. Estos resultados se ratifican con lo manifestado por los estudiantes de los grupos focales, quienes expresaron que las historietas de ánime, los libros de autoayuda o de superación personal y las noticias en la web son sus lecturas favoritas. De cualquier modo, si se tiene en cuenta que la lectura recreativa es aquella que genera goce y se realiza por gusto e iniciativa propia, es sorpresivo que tan solo un 3,8\% le dedique más de 4 horas diarias. 
Ahora bien, el $16,8 \%$ dedica entre 2 y 3 horas diarias a la lectura académica, mientras que el 11,8\% dedica este mismo tiempo a la lectura recreativa. Es posible que esta leve ventaja de la lectura académica se deba al carácter de obligatoriedad, ya que, aunque no sea de su total agrado, los estudiantes cumplen con ella para no ser mal evaluados. Solo el 3,8\% dedica más de 4 horas diarias a la lectura académica, lo que también es síntoma de la poca práctica lectora de los estudiantes que ingresan a la educación superior. Cabe resaltar, además, que varios de los estudiantes expresaron en los grupos focales que no consideraban la lectura como una actividad recreativa, sino como una obligación, lo cual permite vislumbrar la actitud hacia la lectura que estos han forjado a lo largo de la educación primaria y secundaria. Sumado a ello, algunos afirman que la "pereza" y el acceso continuo a las redes sociales son los factores que más influyen negativamente en la formación de un verdadero hábito lector.

Ante las preguntas al grupo focal: “¿Qué tipo de actividades recreativas hacen en su tiempo libre? y ¿la lectura está entre ellas?", los estudiantes mencionaron distintas actividades antes que la lectura: "Yo no es que lea mucho. En mi actividad libre escucho música, salgo con mis amigos y, de vez en cuando, leo cuando me siento como... vago, cojo un libro o algún artículo de lo que sea de mi interés" (participante masculino, grupo focal 2, 18 de mayo de 2017).

En cuanto al lugar de preferencia, se observó que los jóvenes prefieren leer en espacios y condiciones que les son familiares, en tanto que el $65,3 \%$ de los estudiantes manifestó que prefiere leer en casay tan solo un 9,2\% prefiere hacerlo en la escuela o universidad. Un 49,6\% manifiesta no hacer distinción entre el tipo de medio que utilizan para leer, es decir, que pueden realizar lecturas en medios digitales e impresos de manera indistinta. Aspecto que resulta ventajoso a la hora de proponer estrategias de lectura que motiven a los estudiantes. Por último, un $34 \%$ dice que prefiere leer en medios impresos y solo un $16,4 \%$ prefiere medios digitales.

\section{¿Cómo está el hábito lector?}

A partir de los resultados se deduce la presencia de un hábito lector incipiente, es decir, los estudiantes suelen leer de manera esporádica y no siempre manifiestan disfrute de la lectura. Así, el 74,8\% responden al perfil de "lectores no autónomos", que leen solo para cumplir con las tareas asignadas y no por interés propio. Esta tendencia se ratificó durante las charlas con los grupos focales, donde los estudiantes expresaron abiertamente que suelen asumir la lectura como una rutina $u$ obligación. Por ejemplo, una estudiante, cuyo hábito lector fue fomentado por la madre mediante libros de temática religiosa expresó: "mi lectura de la noche de la Biblia la tomo como una rutina" (participante femenino, grupo focal 1, 18 de mayo de 2017). Por su tono de voz y su disposición corporal al expresar su opinión, se confirma que su actividad lectora comporta periodicidad, pero no automotivación, lo que no puede tomarse como un hábito lector en sentido estricto.

Solo un 6,9\% de los estudiantes corresponde al perfil de "no lector", categoría en la que se incluyen aquellos que no practican la lectura como hobbie o como actividad recreativa; no realizan lecturas en su tiempo libre; hacen pocas lecturas diferentes de las suministradas por el docente; no les llama la atención participar en clubes de lectura, y no realizan lecturas de su propio interés. Ahora, si bien el porcentaje de no lectores es bajo, el porcentaje de "lectores autónomos" también lo es (18,3\%).

También se observó que los programas académicos a los que ingresa mayor número de lectores no autónomos son Tecnología en Sistemas de Gestión de Calidad, con el 89,5\%; Administración de Empresas Turísticas y Hoteleras, con 85,3\%; Derecho, con $81,5 \%$; Ingeniería Industrial, con 80\%, y Administración de Empresas, con 79,3\%.

Por otro lado, además de la autonomía, un lector debe echar mano de la recursividad, es decir, debe ser capaz de conseguir el material de lectura 
que desea y que necesita para leer. Con respecto a este factor, en la Figura 2 se observa que, de los estudiantes encuestados, son más los que no se preocu- pan por conseguir el material de lectura requerido, aunque el porcentaje de estudiantes recursivos no es significativamente inferior.

Figura 1. Autonomía lectora. Dimensión Hábitos

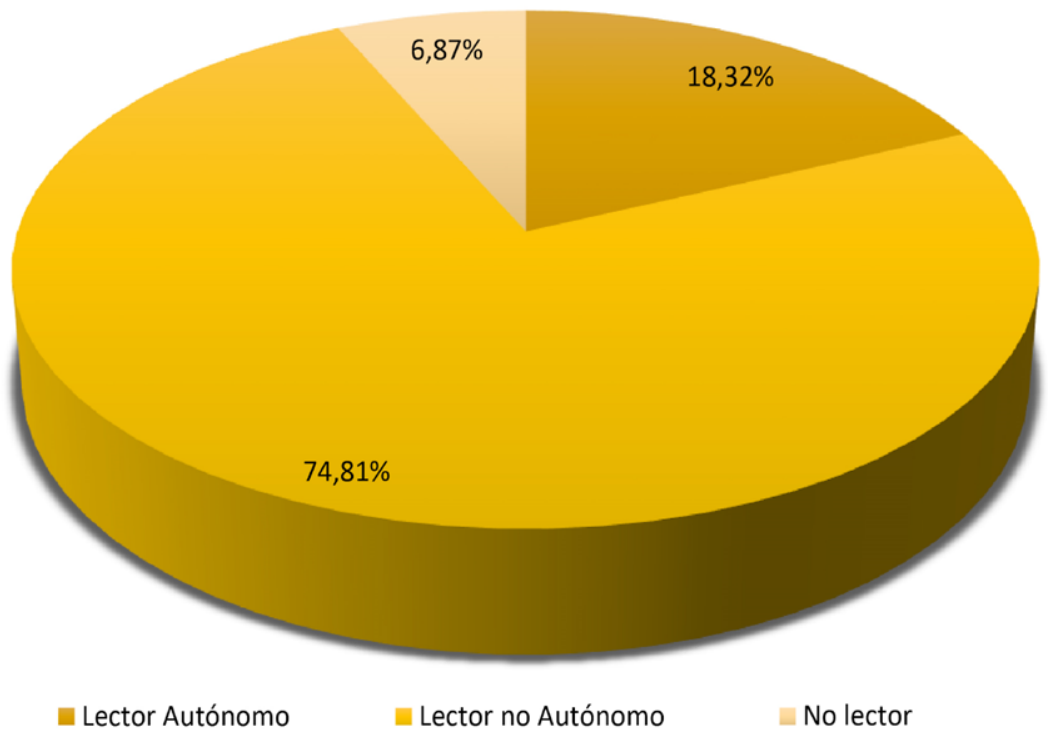

Fuente: aplicación Test de Autorreporte HACES.

Figura 2. Recursividad. Dimensión Hábitos

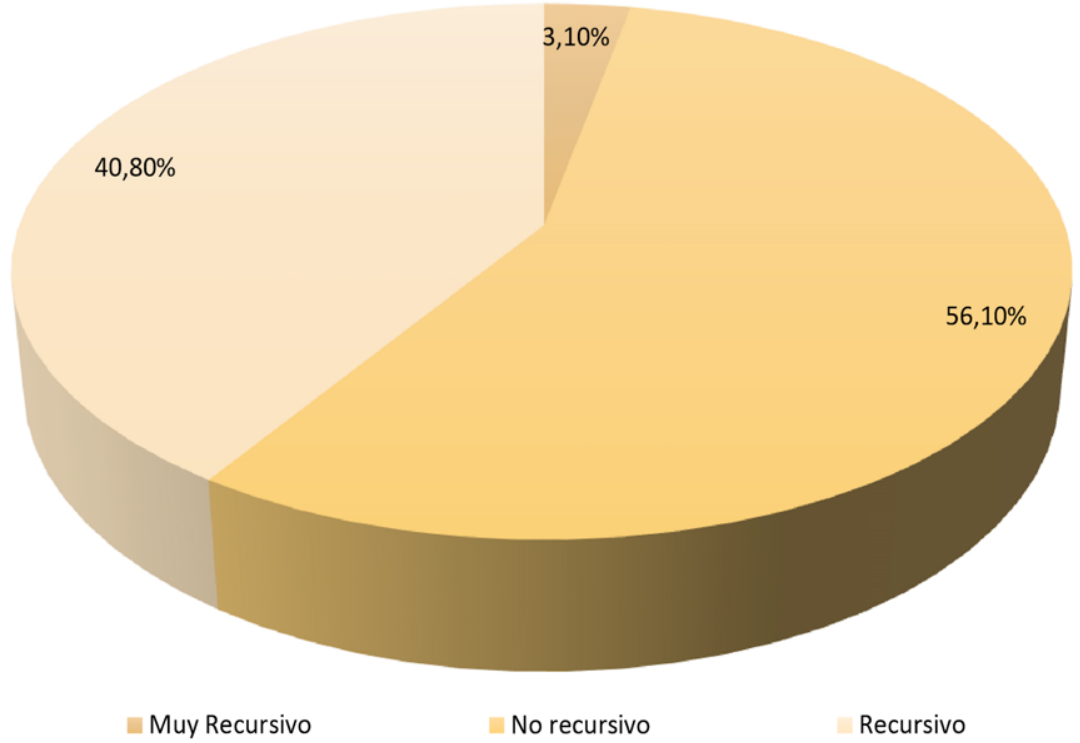

Fuente: aplicación Test de Autorreporte HACES. 
Los estudiantes manifestaron diversos modos de acceder a los libros o materiales de lectura que desean o necesitan: obsequios de familiares, préstamos a un amigo, aplicaciones del celular, navegación en la web y redes sociales. Llama la atención que ninguno manifestó hacer préstamos en una biblioteca. Ahora bien, cabe aclarar que, si bien la recursividad ayuda a hacer más efectivo el hábito, por sí sola no es un indicador de que el sujeto posea un hábito lector. En esa medida, es posible encontrar una persona que, aunque sabe cómo conseguir los recursos y materiales de lectura, lo haga solo por obligación y no porque en realidad ame leer.

Por otra parte, el hábito lector y la actitud de los padres hacia la lectura se traducen en la adquisición del gusto y el desarrollo de conductas lectoras por parte de los hijos (Gil, 2009), lo cual se ratifica en este estudio, puesto que los estudiantes que dicen poseer un hábito lector manifiestan haberlo adquirido gracias al impulso ofrecido en la familia, donde padres o abuelos les proporcionaban libros u otro material de lectura: "Creo que sí tengo un hábito lector, puesto que, desde chiquita, en mi familia me inculcaron de pronto ese..., valga la redundancia, ese hábito lector. Pues sí, mi abuelo me regalaba libros desde chiquita y eso fue como creando ese amor por la lectura" (participante femenino, grupo focal 2, 19 de mayo de 2017).

Otros estudiantes, por su parte, reconocieron que les gustaría adquirir un hábito lector, pero les angustia enfrentarse a las dificultades para comprender un texto y ante la constancia que requiere ser un buen lector.

\section{Actitud hacia la lectura}

En la dimensión Actitudes se observó que los estudiantes poseen una actitud muy positiva cuando se trata de determinar la utilidad de la lectura para la vida académica y social, lo cual evidencia (como se nota en la Tabla 7) que estos reconocen la importancia de la lectura en el proceso de aprendizaje, así como su aporte para el desarrollo intelectual y personal.

Tabla 7. Utilidad de la lectura

\begin{tabular}{|c|c|c|}
\hline İtem & Negativo & Positivo \\
\hline La lectura es útil en mi proceso de aprendizaje & $2,7 \%$ & $97,3 \%$ \\
\hline La lectura es útil en mi proceso de formación personal & $5,0 \%$ & $95,0 \%$ \\
\hline $\begin{array}{l}\text { La lectura es útil para la vida porque desarrolla las habilidades de pensamiento } \\
\text { (sintetizar, interpretar y argumentar) }\end{array}$ & $1,1 \%$ & $98,9 \%$ \\
\hline El ejercicio de la lectura amplía mi vocabulario & $1,1 \%$ & $98,9 \%$ \\
\hline La lectura enriquece mis conocimientos & $2,3 \%$ & $97,7 \%$ \\
\hline Leer me sirve para ampliar mi comprensión del mundo & $5,7 \%$ & $94,3 \%$ \\
\hline
\end{tabular}

Fuente: elaboración propia. 
A pesar de la actitud positiva hacia la lectura académica arrojada por el test, en las sesiones de grupo focal los estudiantes manifestaron cierto grado de aversión o tedio hacia este tipo de lectura, lo cual deja entrever que la actitud positiva hacia la utilidad de la lectura académica no conduce necesariamente al disfrute de la misma: "A mí me impide leer, pues, textos que, la verdad, no me interesan, que sean textos académicos. Me gusta leer cosas de maquillaje, de moda, de películas, novelas, algo de terror, pero cosas ya académicas sí me cuesta mucho leer" (participante femenino, grupo focal 2, 19 de mayo de 2017). Se puede concluir, entonces, que el estar de acuerdo con la importancia de la lectura en la formación profesional no determina la práctica real de la actividad lectora; en otras palabras, un estudiante puede ser consciente de los beneficios de la lectura, aunque en su cotidianidad no la asuma como una práctica habitual: "Sinceramente, a mí no es que me guste mucho la lectura. Sé cuáles son los beneficios de leer, pero no tengo ese hábito de lectura. Me gusta leer artículos que sean de mi entender, por ejemplo, artículos de deportes, o alguna cosa política que me llame la atención" (participante masculino, grupo focal 2, 19 de mayo de 2017).

En cuanto al afecto, los estudiantes encuestados indicaron que leer les genera placer cuando se trata de lecturas recreativas, tal como se había explicado anteriormente. Esta afectividad hacia las lecturas literarias y de entretenimiento de los estudiantes de primer semestre de Unicolombo no está determinada por la escuela de la cual provienen, ya que el $88,7 \%$ de los estudiantes de escuelas privadas y el $86,5 \%$ de escuelas oficiales poseen una afectividad positiva.

Ahora bien, cabe advertir que el gusto por la lectura y el hábito lector son aspectos diferentes. $\mathrm{El}$ gusto se refiere a la simpatía por lo escrito, pero sustentada en poco compromiso y planificación de la actividad lectora, por lo cual se lee de manera esporádica; el hábito, entre tanto, supone un trabajo consciente que requiere voluntad, fijación de objetivos claros y estructuración de procesos mediados (Salazar, 2006). Significa esto, entonces, que es posible encontrar estudiantes que manifiestan un gusto por la lectura, pero que no han forjado un verdadero hábito lector porque no lo hacen con la periodicidad y la avidez que el hábito requiere: "Sé cuáles son los beneficios de leer, pero no tengo ese hábito de lectura, me gusta leer artículos que sean de mi interés, leo solo artículos de deportes, política que me llamen la atención" (participante masculino grupo focal 2,19 de mayo de 2017).

Tabla 8. Afectividad hacia la lectura

\begin{tabular}{|l|c|c|}
\hline \multicolumn{1}{|c|}{ İtem } & Negativo & Positivo \\
\hline La lectura es una actividad placentera para mí & $27,5 \%$ & $72,5 \%$ \\
\hline $\begin{array}{l}\text { La lectura de textos de entretenimiento, como revistas de farándula, } \\
\text { historietas, caricaturas, etc., es divertida }\end{array}$ & $26,3 \%$ & $73,7 \%$ \\
\hline La lectura de cuentos, poemas, novelas, etc., es atractiva & & \\
\end{tabular}

Fuente: elaboración propia. 


\section{Estrategias de lectura utilizadas por los estudiantes}

La mayoría de los estudiantes que componen la muestra hace un uso ocasional de estrategias de comprensión lectora, mientras que los porcentajes de uso frecuente tienden a ser bajos. Solo el 13,4\% usa frecuentemente estrategias de aprestamiento, lo que refleja que pocos estudiantes realizan acciones que permitan una preparación para la lectura; así, hay un número reducido de ellos que explora información general sobre el autor antes de leer (4,2\% lo hace siempre, $11 \%$ lo hace casi siempre). De igual modo, indagar información general acerca del texto antes de leer y establecer con anterioridad el propósito de la lectura también reportan un bajo porcentaje: $8,8 \%$ usa siempre la primera estrategia y $6,5 \%$ usa siempre la segunda. Este panorama es similar al de otras instituciones de educación superior en Colombia. Así, en un estudio realizado en la Universidad de Sucre (Colombia) para medir las estrategias en las fases de prelectura, lectura y poslectura, se evidenció que en la primera fase los estudiantes no activan conocimientos previos y no los relacionan con la nueva información, y tampoco hacen predicciones (Guerra y Forero, 2015, p. 45).

Las estrategias de inmersión, es decir, aquellas que se realizan durante la lectura para reconocer la información principal, son unas de las más usadas por los estudiantes que ingresan a la universidad, ya que un $38,2 \%$ la usa de modo frecuente. Al observar los resultados de cada ítem que conforma este factor, se encontró que el 50,4\% de los estudiantes resalta las palabras claves del texto y el $41,2 \%$ subraya las oraciones principales mientras lee, situación que se corroboró en el diálogo con los grupos focales.

Con respecto alas estrategias de procesamiento de la información, se encontró que el 38,5\% de los estudiantes las usa de manera frecuente y el 58,4\% de manera ocasional. Esto demuestra que, durante la lectura, los estudiantes buscan en el diccionario las palabras que desconocen; se detienen al encontrar ideas dificiles de comprender y releen para hallar el sentido; así mismo, consultan a otra persona cuando encuentra frases difíciles de comprender, piden la opinión de alguien más e incluso buscan videos en la web que explican la temática tratada.

\section{Figura 3. Uso de estrategias de lectura}

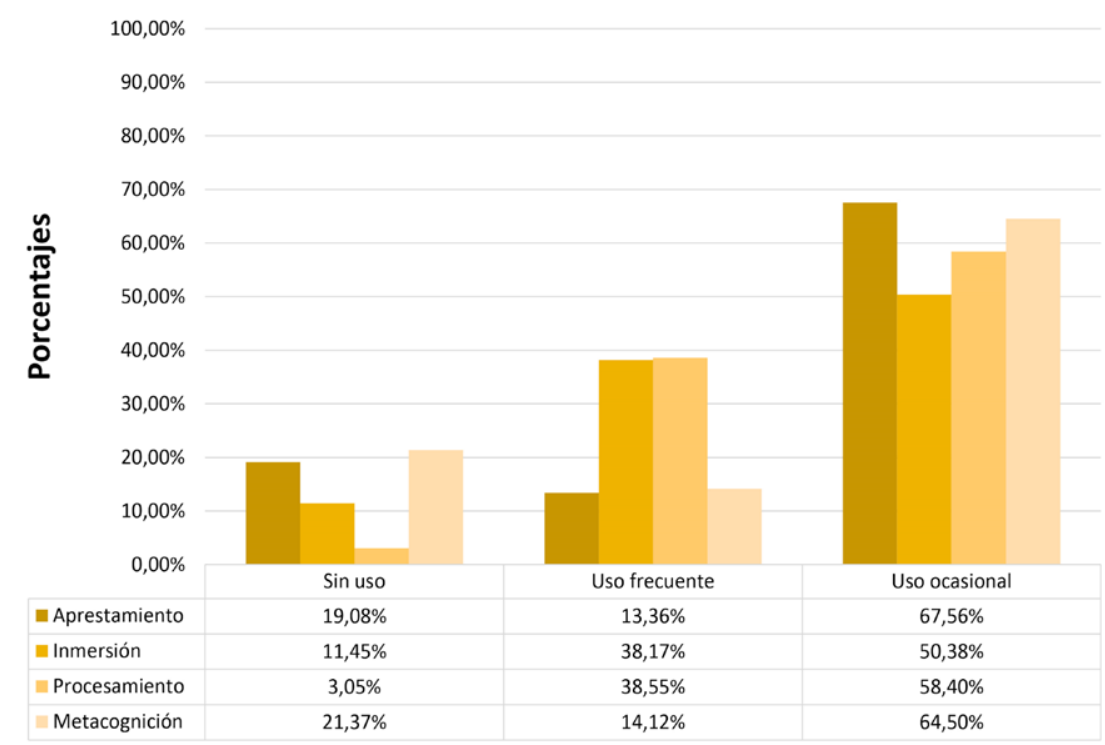

Fuente: aplicación Test de Autorreporte HACES. 
Los estudiantes que ingresan al programa de Derecho son los que usan con mayor frecuencia las estrategias de aprestamiento de la lectura (25,7\%); los que ingresan al programa de Licenciatura en Bilingüismo con énfasis en Inglés son los que más usan las estrategias de inmersión a la lectura (20\% de uso frecuente) y las estrategias de metacognición (21,6\% de uso frecuente). En cuanto al uso de las estrategias de procesamiento de la información, todos los programas presentan un porcentaje similar, salvo Tecnología en Gestión de Servicios Turísticos y Hoteleros, que reportó un alto porcentaje de no uso de este tipo de estrategia (50\%). Llama la atención que los estudiantes de este último programa son los que reportan un porcentaje más alto en el no uso de las estrategias de aprestamiento (32\% de no uso), de inmersión (33,3\%) y de metacognición $(28,6 \%)$. Corresponde entonces a este programa y a la institución pensar en acciones pedagógicas que apunten al mejoramiento de este panorama.

Finalmente, en cuanto a las estrategias de metacognición, el 21,4\% de los estudiantes manifestó no usarlas y solo el 14,1\% las usa frecuentemente, porcentajes que también preocupan, en la medida en que evidencian una falta de interés por regular el proceso mismo de comprensión y garantizar una buena interpretación. De acuerdo con Mokhtari y Reichard (2002) y Jiménez (2004), dentro de estas estrategias se incluyen: realizar apuntes durante la lectura; elaborar resúmenes después de la lectura; elaborar esquemas y mapas conceptuales; buscar información en otras fuentes para ampliar lo leído, y formular preguntas después de lo leído para comprobar si se entendió. Resultados similares encontraron Ramírez, Marín, Ospino y Meneses (2008) en su investigación sobre estrategias de lectura en la educación superior. Allí evidenciaron que entre las estrategias más usadas por los estudiantes de primer semestre de Medicina están los apuntes para repasar, resaltar palabras clave y subrayar las oraciones principales de cada párrafo, mientras que poco se utiliza la elaboración de cuadros sinópticos y mapas conceptuales. Guerra y
Forero (2015) hallaron en su estudio en la Universidad de Sucre que durante la fase de poslectura entre un $50 \%$ y un $76 \%$ no identificó la idea principal; no realizó el resumen; no relacionó el texto leído con sus vivencias, con otros textos y otros contextos, y tampoco sintetizó la información en un organizador gráfico, y solo el $21 \%$ relacionó lo leído con sus vivencias.

\section{Discusión}

El porcentaje de lectores autónomos es bajo, situación que se torna preocupante, en la medida en que se espera que los estudiantes que ingresan a la educación superior posean un interés genuino por la lectura. Según lo manifestado en los grupos focales respecto al acceso a diversas formas de entretenimiento, se puede afirmar que entre los jóvenes estudiados hay un escaso gusto por la lectura debido a la preferencia por otro tipo de actividades de recreación.

En la mayoría de los programas académicos que ofrece la institución ingresaron estudiantes que hacen parte de la categoría de no lectores, lo cual evidencia que los jóvenes ingresan con un hábito lector débil, independientemente de la carrera a la cual se dirijan, lo que implica redoblar los esfuerzos por parte de la institución para que estos alcancen un nivel adecuado de competencia comunicativa.

Se observa también que la mayoría de los jóvenes tiende a leer por asignación de terceros y no por iniciativa propia, y esta tendencia es la misma para estudiantes provenientes tanto de escuelas oficiales (75\% son lectores no autónomos) como de escuelas privadas (74,2\%). Estos resultados son similares a los hallados por Navas y García (2009), quienes, a partir de su proyecto de fomento a la lectura en una facultad de Lenguas en México, concluyeron que el hábito de lectura desarrollado por los estudiantes universitarios no es una actividad relacionada con su vida diaria, sino una tarea que realizan fundamentalmente como parte del quehacer académico. Granado y Puig (2014) también observaron una rela- 
ción "tibia y utilitaria" con la lectura, puesto que la mayor parte de las lecturas evocadas por los jóvenes estudiantes de profesorado son lecturas obligatorias realizadas por exigencia de sus estudios. Larrañaga y Yubero (2005), por su parte, consideran que el estudiante universitario "no lector" lee con poca frecuencia y orientado siempre hacia la lectura obligatoria, su acceso a los libros es por necesidad y es probable que abandone la lectura cuando finalice su periodo de formación universitaria.

Si bien se manifiesta una actitud positiva en cuanto a la utilidad de la lectura, es poca la motivación para desarrollar un verdadero hábito lector. Dicha situación no es exclusiva de la población estudiada, sino que también se evidencia en otros contextos universitarios, a nivel tanto nacional como internacional. Tal como señaló Araújo (2006), un gran número de alumnos ingresan a la universidad sin haber adquirido las competencias básicas de lectura y escritura de textos complejos ni la capacidad para el razonamiento lógico, sintesis, análisis y argumentación.

Los resultados anteriores indican que los estudiantes que ingresan a la Fundación Universitaria Colombo Internacional necesitan reforzar sus competencias en lectura y escritura para afrontar las actividades académicas que involucra el plan de estudios de cada programa y para cumplir con las competencias genéricas y específicas exigidas a todos los profesionales. Aquí vale la pena señalar que es importante fomentar entre los jóvenes la idea de que el hábito lector no está reservado a estudiantes con capacidades excepcionales, sino que cualquier sujeto es susceptible de adquirirlo, toda vez que lo haga consciente y se esfuerce en la práctica.

En las instituciones universitarias se suelen realizar prácticas pedagógicas partiendo de la idealización del rol del estudiante, es decir, se parte de la presunción de que este ya ha desarrollado las competencias básicas de lectura y escritura durante la educación media, olvidando que a la universidad también le corresponde la labor de continuar con el afianzamiento progresivo de la competencia lectora. La asignación de lecturas de textos extensos y complejos sin hacer seguimiento al proceso de comprensión, y la exigencia de resultados inmediatos sin ofrecer las condiciones de tiempo o espacio para desarrollar la lectura provocan entre los jóvenes ingresantes una atmósfera de ansiedad y temor que los lleva a reforzar su animadversión.

Lo anterior conlleva la necesidad de repensar las prácticas pedagógicas en la enseñanza de la lectura en la educación universitaria y particularmente en la institución estudiada ${ }^{2}$. Se requiere que los docentes de todas las áreas disciplinares agencien prácticas de lectura más significativas, partiendo del conocimiento de las percepciones o actitudes de los estudiantes frente a la lectura, así como de los hábitos y las estrategias que ponen en juego a la hora de leer. El acostumbramiento de los estudiantes a la lectura de textos académicos propios de su disciplina debe ser progresivo y guiado, aún más si se tienen en cuenta las dificultades que ya presentan. La educación superior debe asegurar que los estudiantes comprendan la importancia de adoptar una actitud más positiva y de asumir el proceso de lectura con un mayor nivel de conciencia para ser lectores competentes.

\section{Conclusiones}

Al ingresar a la universidad, el estudiante se integra a una nueva cultura académica con códigos y lógicas distintas de la escolar (Muñoz, Valenzuela, Avendaño y Núñez, 2016), por lo cual la transición de la escuela a la educación superior puede

2 Cabe señalar que a partir de esta investigación se realizó un ajuste a los microcurrículos de las asignaturas Competencias Comunicativas I y II (Licenciatura en Bilingüismo) y Comunicación y Lenguaje I y || (Administración de Empresas) para incluir una unidad inicial de comprensión lectora con que afianzar las habilidades asociadas a los niveles literal, inferencial y crítico, y ofrecer a los estudiantes estrategias de lectura que les permitan elevar el nivel de comprensión de los textos académicos. De igual modo, a partir del segundo periodo de 2017 , se dio apertura -en su fase de prueba piloto- al Centro de Lectura y Escritura. La efectividad de estos planes será medida en un estudio posterior. 
tornarse difícil, puesto que esta nueva cultura académica involucra ciertos tipos de texto que exigen habilidades para inferir, interpretar, reflexionar y evaluar la información, más allá de la localización de información literal (Pereda, Cereceda, y Martínez, 2015). En la vida universitaria los estudiantes se enfrentan al aprendizaje sistemático de los recursos lingüísticos y discursivos propios de la comunicación especializada (Castro y Camargo, 2016), a los que posiblemente no tuvieron acceso en los niveles educativos anteriores.

Se requiere entonces del esfuerzo por parte del cuerpo profesoral para hacer acompañamiento en los procesos de lectura de los estudiantes de semestres iniciales y se precisa también, al decir de Olave, Rojas y Cisneros (2013), trascender las viejas prácticas de lectura, para evitar que el estudiante pueda sufrir una desadaptación que lo conduzca a una deserción temprana.

Es necesaria la intervención de los docentes explícitamente dirigida a la adquisición de adecuadas estrategias de lectura para comprender los textos (Solé, 1998). Dichas estrategias deben enseñarse en el contexto de las asignaturas, para que favorezcan el aprendizaje del estudiante en su campo disciplinar, puesto que,enseñadas de manera descontextualizada, impiden quelos estudiantes aprendan cómo aplicarlas en las áreas de conocimiento que necesitan abordar (Zanotto, 2016). Explicitar el objetivo de la lectura, ofrecer información general acerca del texto y del autor son estrategias de aprestamiento básicas que permiten allanar el camino para el proceso de interpretación, y que deben ser puestas en práctica de manera conjunta en el aula.

Para incentivar la motivación por la lectura no basta con partir del axioma general de que leer es bueno y necesario, sino que se debe presentar cada texto de manera tal que se visibilice el porqué y el para qué de la lectura (Aragón y Arias, 2010). Adicionalmente, para que los estudiantes sean autónomos al momento de enfrentarse a los distintos tipos de textos, deben conocer los procesos implicados en la comprensión y cómo regularlos a través de estrategias cognitivas y metacognitivas que deben ser puestas en marcha antes, durante y después de la lectura (Pardo, 2015).

Para abordar de manera integral la lectura es preciso adelantar acciones que trasciendan los límites de una única asignatura. Las asignaturas de lectoescritura prescritas en los primeros semestres suelen ofrecer entrenamiento con respecto a la acentuación, puntuación, ortografía, coherencia, cohesión, distinción entre géneros discursivos, etc., elementos importantes para la corrección lingüística al escribir, pero que no son suficientes para garantizar la alfabetización académica de los estudiantes, puesto que se enfocan en las habilidades de tipo genérico y no en las particularidades enunciativas y discursivas de cada ciencia o disciplina.

Por último, es preciso modificar algunas de las concepciones acerca de la lectura, puesto que asumirla solo con un fin instrumental desdibuja su función cognitiva o epistémica y su capacidad de dotar al lector de pensamiento crítico. Se debe, entonces, diseñar un plan de lectura que propicie el trabajo colaborativo (pues la lectura es una actividad que requiere de asistencia y acompañamiento) y que estimule la metacognición y el uso de estrategias de pensamiento reflexivo (Rivera, 2003; Díaz y Hernández, 2010).

En suma, la formación universitaria debe estar enfocada en el fortalecimiento de la alfabetización académica que permita a todo profesional tener la capacidad de leer textos propios de su disciplina, y de distinta naturaleza, con sentido crítico. Por ello, todo el cuerpo profesoral de la institución estudiada debe hacerse consciente de la responsabilidad que tiene la universidad de dar continuidad a la enseñanza de la lectura y de dotar a los estudiantes de estrategias que permitan forjar nuevos hábitos y actitudes más positivas frente a ella. 
ISSN 0123-1294 | e-ISSN 2027-5358 | Educ.Educ. Vol. 21. No.3 | Septiembre-diciembre de 2018 | pp. 482-503.

Universidad de La Sabana | Facultad de Educación

\section{Referencias}

Aragón, R. B. y Arias, Y. M. (2013). La lectura: actitudes y motivaciones de los estudiantes de primer semestre de la Fundación Universitaria del Área Andina. Revista Fundación Universitaria del Área Andina, 13, 58-76. Recuperado de http://revia.areandina.edu.co/ojs/index.php/Gg/article/view/343

Araújo, J. (2006). Articulación Universidad-Escuela Media. Política para la definición de competencias para el acceso a la educación superior. Documento CPRES-Secretaría de Politicas Universitarias, Ministerio de Educación, Ciencia y Tecnología, Argentina.

Arista, S.M.y Paca, N.K. (2015). Los hábitos de lectura y la comprensión lectora en estudiantes universitarios de la especialidad de Lengua, Literatura, Psicología y Filosofía en el año 2014. Revista de Investigación Altoandina, 17(3), 379-386. DOI: 10.18271/ria.2015.137

Beneitone, P., Esquetini, C., González, J., Marty, M., Siufi, G. y Wagenaar, R. (2007). Reflexiones y perspectivas de la Educación Superior en América Latina. Informe final proyecto Tuning América Latina 2004-2007. Bilbao: Universidad Deusto.

Calderón, A. y Quijano, J. (2010). Características de comprensión lectora en estudiantes universitarios. Revista Estudios Socio-Jurídicos, 12(1), 337-364. Recuperado de https://revistas.urosario.edu.co/index.php/sociojuridicos/article/view/1195

Carlino, P. (2013). Alfabetización académica. Diez años después. Revista Mexicana de Investigación Educativa,18(57), 335-381. Recuperado de https://www.redaly c.org/pdf/140/14025774003.pdf

Carlino, P.y Fernández, G. (2010). ¿En qué se diferencian las prácticas de lectura y escritura de la universidad y las de la escuela secundaria? Lectura y Vida, 31(3), 6-19. Recuperado de https://media.utp.edu.co/referenciasbibliograficas/uploads/referencias/articulo/fernandezy carlinozo1oenquesediferencianpdf-k6l7l-articulo.pdf

Castro, M. y Camargo, M. (2016). Características genéricas y estrategias de lectura. Una propuesta para la comprensión de textos académicos. En G. Bañales, M. Castelló y N. Vega (coords.), Enseñar a leer y escribir en la educación superior. Propuestas educativas basadas en la investigación (pp. 77-100). Recuperado de http://www. fundacion-sm.org.mx/sites/default/files/Ense\%C3\%B1ar\%20a\%2oleer\%20y\%2oescribir.pdf

Cerlalc-Unesco (2011).Metodología común para explorary medir el comportamiento lector. Bogotá: Cerlalc. Recuperado de https://cerlalc.org/wp-content/uploads/publicaciones/olb/PUBLICACIONES_OLB_\%2O Metodologia-comun-para-explorar-y-medir-el-comportamiento-lector_v1_010111.pdf

Cerlalc-Unesco (2012). Comportamiento lectory hábitos de lectura. Una comparación de resultados en algunos países de América Latina. Recuperado de http://cerlalc.org/comportamiento-lector-y-habitos-de-lectura-3/

Conradi, K., Jang, B. G., Bryant, C. L., Craft, A.y McKenna, M. (2013). Measuring Adolescents' Attitudes Toward Reading. A classroom Survey. Journal of Adolescent \& Adult Literacy, 56(7), 565-576. DOI: 10.1002/JAAL.183 
Díaz, F.y Hernández, G. (2010). Estrategias docentes para un aprendizaje significativo. Una interpretación constructivista. México: McGraw-Hill.

Gil, J. (2009). Hábitos y actitudes de las familias hacia la lectura y competencias básicas del alumnado. Revista de Educación, 350, 301-322. Recuperado de http://www.revistaeducacion.mec.es/re350/re350_13.pdf

Gilardoni, C. (2006). Universitarios y lectura: Análisis cuali-cuantitativo del uso, accesibilidad y valoración de los libros. Calidad en la Educación, 25, 213-239. DOI: 10.31619/caledu.n25.260

Gordillo, A. (2015). Las tendencias de la lectura y la escritura en la educación superior en Colombia. En C. Muse (ed.), Lectura y escritura en el nivel superior (pp. 310-314). Córdoba: Universidad Nacional de Córdoba.

Granado, C. y Puig, M. (2014). ¿Qué leen los futuros maestros y maestras? Un estudio del docente como sujeto lector a través de los títulos de libros que evocan. Ocnos, 11, 93-112. DOI: 10.18239/ocnos_2014.11.05

Guerra E.y Forero, C. (2015). Estrategias para el desarrollo de la comprensión de textos académicos. Zona Próxima, 22, 33-35. Recuperado de https://www.redaly c.org/articulo.oa?id=85339658004

Hernández-Sampieri, R., Fernández, C. y Baptista, P. (2014). Metodología de la investigación. México: MacGraw-Hill.

Jiménez-Rodríguez, V. (2004). Metacognición y comprensión de la lectura: evaluación de los componentes estratégicos (procesos y variables) mediante la elaboración de una escala de conciencia lectora (Escola). Tesis doctoral, Universidad Complutense de Madrid.

Larrañaga, E. y Yubero, S. (2005). El hábito lector como actitud: El origen de la categoría de "falsos lectores". Ocnos, 1, 43-60. DOI: 10.18239/ocnos_2005.01.04

McKenna, M. y Kear, D. (1990). Measuring attitude toward reading: A new tool for teachers. The Reading Teacher, 43(8), 626-639. DOI: 10.1598/RT.43.8.3

Mezzalira, M. y Boruchovitch, E. (2016). Escala de Motivação para a Leitura para Adolescentes e Jovens: Propriedades Psicométricas. Psicologia:Teoria e Pesquisa, 32(2), 1-9. DOI: 10.1590/0102-3772e32227

Ministerio de Educación Nacional (2017). Marco de Referencia Preliminar para Competencia Lectora. Bogotá: Icfes.

Molina, V. (2008). ¿Es que los estudiantes no leen ni escriben?: El reto de la lectura y la escritura en la Pontificia Universidad Javeriana. En H. Mondragón (ed.), Leer, comprender, debatir, escribir. Escritura de artículos cientificos por profesores universitarios (pp. 53-62). Cali: Sello Editorial Javeriano.

Mokhtari, K. y Reichard, C. (2002). Assessing Students' Metacognitive Awareness of Reading Strategies. Journal of Educational Psychology, 94(2), 249-259. DOI: 10.1037/0022-0663.94.2.249

Mokhtari, K., Reichard, C. y Dimitrov, D. (2018). Revising the Metacognitive Awareness of Reading Strategies Inventory (MARSI) and testing for factorial invariance. Studies in Second Language Learning and Teaching, 8(2), 219-246. DOI: 10.14746/ssllt.2018.8.2.3 
Muñoz, C., Valenzuela J., Avendaño, C. y Núñez, C. (2016). Mejora en la motivación por la lectura académica: la mirada de estudiantes motivados. Ocnos, 15(1), 52-68. DOI:10.18239/ocnos_2016.15.1.941

Nava, G. y García, C. (2009). Análisis de la cultura lectora en estudiantes universitarios. Educación, 33(1), 42-59. Recuperado de https://www.redaly c.org/articulo.oa?id=44015082004

Olave-Arias, G., Rojas-García, I. y Cisneros-Estupiñán, M. (2013). Deserción universitaria y alfabetización académica. Educación y Educadores, 16(3), 455-471. DOI: 10.5294/edu.2013.16.3.4

Parales-Quenza, C. y Vizcaíno-Gutiérrez, M. (2007). Las relaciones entre actitudes y representaciones sociales: elementos para una integración conceptual. Revista Latinoamericana de Psicología, 39(2), 351-361. DOI: 10.14349/rlp.v39i2.327

Pardo, N. (2015). Estrategias autorreguladoras para la comprensión de textos académicos en los estudiantes universitarios. Horizontes Pedagógicos, 17(2), 29-38. Recuperado de https://revistas.iberoamericana. edu.co/index.php/rhpedagogicos/article/view/902

Pereda, M., Cereceda, C. y Martínez, J. (2015). Lectoescritura: literacidad y comunidad epistémica. En C. Muse (ed.), Lectura y escritura en el nivel superior (pp. 324-336). Córdoba: Universidad Nacional de Córdoba.

Pérez Abril, M. y Rincón Bonilla, G. (2013). ¿Para qué se lee y se escribe en la universidad colombiana? Un aporte a la consolidación académica del país. Bogotá: Pontificia Universidad Javeriana.

Petscher, Y. (2009). A meta-analysis of the relationship between student attitudes towards reading and achievement in reading. Journal of Research in Reading, 33(4), 335-355. DOI: 10.1111/j.1467-9817.2009.01418.x

Ramírez, A., Marín, C., Ospino, L. C., Meneses, G. (2008). Estrategias lectoras en educación superior. Memorias. Revista de Investigaciones, 3(10), 66-76. Recuperado de https://media.utp.edu.co/referencias-bibliograficas/uploads/referencias/ponencia/1036-estrategias-lectoras-en-educacion-superiorpdf-R68Wv-articulo.pdf

Rivera, M. (2003). Estrategias de lectura para la comprensión de textos escritos: el pensamiento reflexivo y no lineal en alumnos de educación superior. Revista Digital Umbral, 12, 1-14. Recuperado de https://media. utp.edu.co/referencias-bibliograficas/uploads/referencias/ponencia/1070-estrategias-de-lectura-para-lacomprension-de-textos-escritos-el-pensamiento-reflexivo-y-no-lineal-en-alumnos-de-educacion-superiorpdf-oPtDd-resumen.pdf

Salazar-Ayllón, S. (2006). Claves para pensar la formación del hábito lector. Allpanchis, 66, 13- 46. Recuperado de http://eprints.rclis.org/8551/

Solé, I. (1998). Estrategias de Lectura. Barcelona: Graó.

Velásquez, J. C. y Calvache, I. (2011). Estudio sobre hábitos de lectura y escritura en el ámbito académico en el programa de zootecnia de la Universidad de La Salle. Ciencia Animal, 4, 25-39. Recuperado de https:// revistas.lasalle.edu.co/index.php/ca/article/view/314 
Wigfield, A., Guthrie, J. y McGouh, K. (1996). A questionnaire measure of children's motivation for reading. Instructional Resource, 22,1-24. Recuperado de https://files.eric.ed.gov/fulltext/ED394137.pdf

Zanotto, M. (2016). Comprensión lectora y aprendizaje de textos académicos: hacia una lectura estratégica en el campo de las ciencias sociales. En G. Bañales, M. Castelló y N. Vega (coords.), Enseñar a leer y escribir en la educación superior. Propuestas educativas basadas en la investigación (pp. 27-51). Recuperado de http:// www.fundacion-sm.org.mx/sites/default/files/Enseñar a leer y escribir.pdf 